\title{
Stability Monitoring and Control of Generation Based on the Synchronized Measurements in Nodes of Its Connection
}

\author{
Alexander Fishov ${ }^{1}$, Maria Shiller ${ }^{2}$, Anton Dekhterev ${ }^{2}$ and Vladimir Fishov ${ }^{2}$ \\ 1. Automated Electrical Power Systems Department, NSTU (Novosibirsk State Technical University), Novosibirsk 630073, Russia \\ 2. Novosibirsk Regional Dispatching Office of System Operator of the United Power System, JSC (Novosibirsk RDO SO UPS, JSC), \\ Novosibirsk 630007, Russia
}

Received: October 04, 2014 / Accepted: November 13, 2014 / Published: January 31, 2015.

\begin{abstract}
Synchronized distributed measurements of mode parameters create a technical feasibility for development and implementing new technologies of control the mode stability and the admissibility of EPS (electric power system) mode. Discussion will focus on different models obtained from data synchronized measurements for operational and automatic emergency control without EPS being totally controlled. According to the proposed technology, the generator's output power restrictions are determined in real-time by the terms a static stability using the generators' mode model as a multipole with connection nodes of generators' electromotive forces (the matrix of SMA (self and mutual admittances) of electromotive forces of generators). Potential applications of the technology are distribution network with the main substation and generators of commensurable capacity, and transmission network with large power plants (generators) distributed into the network. The one-level control system for all of generators with defining the generator's power limits relative to the main substation is implemented in the first case. In the second case, the two-level control system is brought in, based on the separation of large and small generation motion. The results of the method and technology efficiency verification are shown in the paper, by both computer simulations of the power system modes and its physical model.
\end{abstract}

Key words: Power system, static stability, synchronized measurements, normal mode, transient mode, post emergency mode, matrix of self and mutual admittances.

\section{Introduction}

The control of the mode stability during the operation of EPS (electric power system) is essential in the systems of technological and emergency control schemes, operating control for providing stability of the parallel operation of generators either in normal, post emergency mode or in relatively short quasi steady mode, which happens after the immediate attenuation of electromechanical transients.

The currently applied methods of control are based on the usage of mathematical models of the electric

Corresponding author: Alexander Fishov, professor, research fields: power system stability, power system emergency control, and automated control system. E-mail: fishov@ngs.ru. transmission system, which are based on the grid topology and parameters of all of its elements [1].

The integration of the distributed generation into electric transmission systems with large power plants in the area of distribution grid increases the complexity of the problem and considerably complicates a required control system. Furthermore, the efficiency of its usage decreases. This presents the technological barrier to the development of distributed generation and offers an opportunity for new technology to control the stability, which would enable more preferable conditions for integration of distributed generation in the current EPS and grid.

Synchronized distributed measurements of modes' parameters create a technical feasibility for 
development and implementing new technologies of control the mode stability and the acceptability of EPS mode which are able to solve the problem. These measurements enable to obtain models for taking into consideration limits towards stability of EPS mode to the operating and automatic emergency control which suit current mode of the grid but without being totally controlled. Such feasibility is explored in previous works [2-4] as well as in the current works [5-7].

\section{Theoretical Basis}

\subsection{Model of Generators Mode and Control of Some} Network Parameters

The proposed technology of controlling the limits for generators to distribute the capacity by the condition of a static stability in real time is based on the model of mode of generators in terms of multi-port circuit nodes of connection of their electromotive forces (the matrix of SMA (self and mutual admittances) of generator electromotive forces of generators). In initial condition, model does not contain another node of commonly used equivalent circuit of EPS besides nodes of generator EMF. This means that loads of the systems are shown as linear bridges. Counting or miscounting voltage regulation depends on equivalent circuit being used and the way of describing changes of the EMFs.

Let us show electrical mode of EPS generators in the current time period as passive linear multi-port with EMF connected to the nodes.

Mode of $n$ generators is described by system of equations:

$$
\underline{S}=\operatorname{diag}\{\underline{E}\} \cdot \underline{Y}^{*} \cdot \underline{E}^{*}
$$

where, $\underline{S}$ is matrix (vector ) of inside active and reactive powers of generators on the nodes of EMF. $\underline{Y}$ is square matrix of SMA of generator EMFs. $\underline{E}$ is matrix of vectors of generators EMFs.

Mode of each of generators is described as:

$$
\underline{S}_{i}=\underline{E}_{i} \sum_{j=1}^{n} \underline{Y}_{i j}^{*} \underline{E}_{j}^{*} \quad(i=1,2, \ldots, n)
$$

where, $i$ and $j$ are indexes which show number of generators in the scheme. $\underline{S}_{i}$ is inside power of $i$ generator. $\underline{E_{i}}, \underline{Y}_{i i}$ and $\underline{Y}_{i j}$ are elements of matrix $\underline{E}$ and $\underline{Y}$.

Eq. (2) can be written as:

$$
\underline{S}_{i}=\underline{E}_{i} \cdot\left(\underline{E}^{*}\right)^{\mathrm{T}} \cdot \underline{y}^{(\mathbf{i})^{*}}
$$

where, $\underline{y}^{(i)}$ is column matrix (vector) SMA of $i$ generator which conforms to $i$ line of matrix $\underline{Y}$.

Synchronized measurements of active and reactive power, vector measurements of voltage in the nodes of power connection allow for each of the time periods (power modes) calculate matrix $\underline{S}$ and $\underline{E}$.

Rewrite Eq. (3) for $n-1$ generator for one mode and we have more generally undefined system of linear independent equations relative to SMA vector of generators, unit vector $\underline{\mathrm{y}}^{(i)}$ :

$$
\underline{S}=\underline{A} \cdot \underline{y}^{*}
$$

where, $\underline{A}$ is right-angled matrix, where coefficients are producing EMF with index coincide with index of conductivity, though in every line, element with distinct first index of the line are cleared.

For making system of Eq. (4) defined (with square matrix $\underline{A}$ ) or redefined, it should be complimented using measurements for different time periods of transitional mode together with redistribution power between generators.

In the result of solving defined or redefined system of Eq. (4), there are parameters of multi-port (SMA of generator EMFs.)

With necessity of control inside parameters of the grid (voltage in some nodes, power flow in the dedicated section), it should be installed additional units of synchronized measurements in the appropriate nodes of the grid and power lines. By the results of measurement, identification of complex coupling coefficients with EMF of generators and then defining needed controlled parameters in quasi steady and post emergency modes are possible. 


\subsection{The Model Identification}

The necessary condition for actual SMA matrix identification (having system of Eq. (4) without degeneracy) is occurrence of steady state changes (redistribution of power between generators). It is possible by two ways:

The first is by using sporadic disturbance of normal mode of EPS in the result of emergency disturbance. In this case, SMA matrix identifies in the time period of transient mode which is connected with attenuation of electromechanical transients and the application domain is control of acceptability quasi steady and steady PEM (post emergency mode).

The second is by using artificial changes of mode, e.g., by using short time unloading of generator by active power. In that case, there can be provided the control of limits of acceptability of normal mode (limits of producing power by generators).

The sufficient condition of actual SMA matrix identification is a representation of generators that characterized by in-phase rotors motion as the one equivalent generator, so far as in general case in complicated multi-machine EPS, a power redistribution between generators (or power plants) during disturbances or operations occurs between some of generators only. An absence of mode changes of residual generators does not allow identifying their mutual admittances due to a degeneracy of the system of equations. The obtained model in this case shows the structure of the mutual generators motion during the power redistribution between them.

The overdetermination of Eq. (4) is required for stable results under a presence of the measuring error and method error conditioned by the substitution of a real object mode by its specified model. In Fig. 1, the mutual admittances identification results for the scheme containing three generating nodes and infinite buses, are presented. It can be seen from Fig. 1 that the measurements redundancy increase improves the stability of identification results of SMA matrix values.

\subsection{Using the Model for Control}

The model obtained can be used in calculating the limits on the power output of each network generators for the steady state, quasi steady, and post emergency mode taking into account the work of excitation regulators.

During the operating control of the output power of the generators, while operator is controlling the stability with the suggested technology, several actions should be performed. Firstly, the operator reduces the

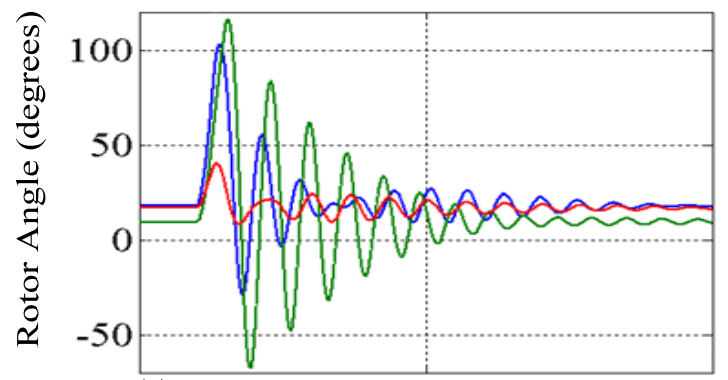

(a)

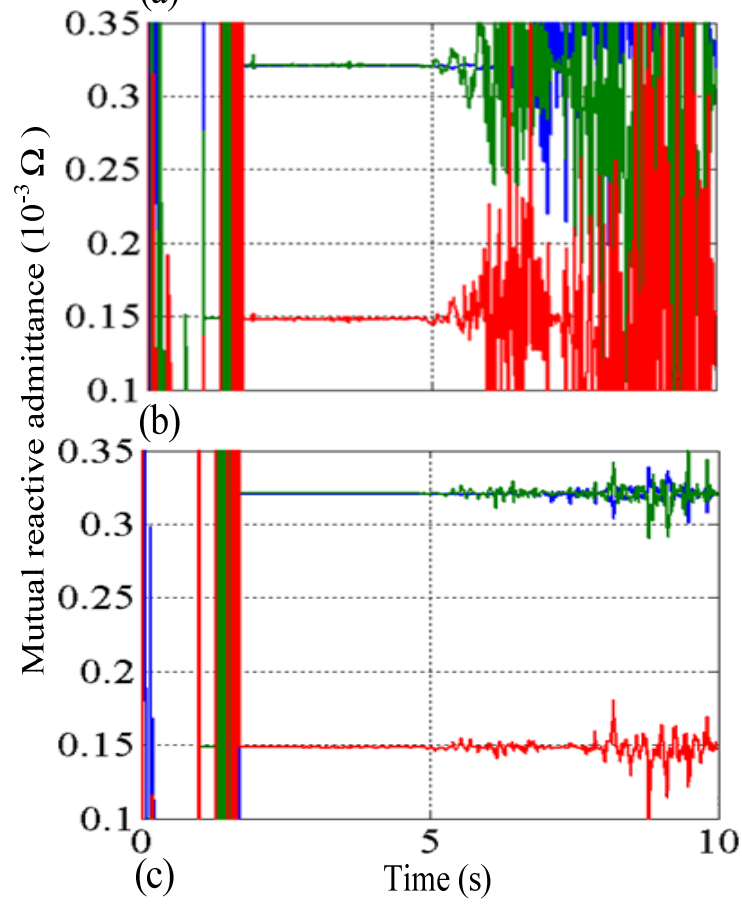

Fig. 1 The equations set overdetermination impact on the stability of identification results of the SMA matrix during electromechanical transients after disturbance (identification "window" is 3-5 s): (a) rotor angle oscillations of generators; (b) mutual reactive admittance from SMA matrix subject to the fourfold overdetermination; and (c) mutual reactive admittance from SMA matrix subject to the tenfold overdetermination. 
generator's output power by some small quantity, which is sufficient for identification of SMA matrix during the redistribution of power in the system. After the procedure of identification and definition of the generator's output power, restrictions were done, then, the desired change of the generation mode can be realized. Furthermore, during the process of changing, the control of the restrictions in real time continues (Fig. 2).

If unexpected changes occur in normal network conditions, also the power output limits are defined to be used, if necessary, to prevent violations of stability automatically or manually. Specifically, in respect to control of turbine power to provide the required stability margin in post-emergency mode, it is can be changed the level of long-time turbine off-loading. This will improve the controllability of EPS and reduce redundancy control actions.

The structure of restrictions control system for different purposes networks has the features:

(1) Distribution network with supply center (node connection to the main network) and generators in different nodes. In this case, a single-level control system is implemented for all generators of the distribution network. Limit power of each generator are determined to direction of weighting generator-supply center.

(2) Transmission network EPS containing large power stations (generators) and on small nodes distributed generation. In this case, two-level control system is implemented on the basis of separation of motions large and small generation. Parallel work stability of large generators is provided by traditional technology. Stability areas with distributed generation, according to the proposed technology under controlled conditions movement of large generators.

\subsection{Advantages and Peculiarities of the Method}

The proposed method assumes the stability control in node coordinates (generators active power coordinates), that provides following advantages:

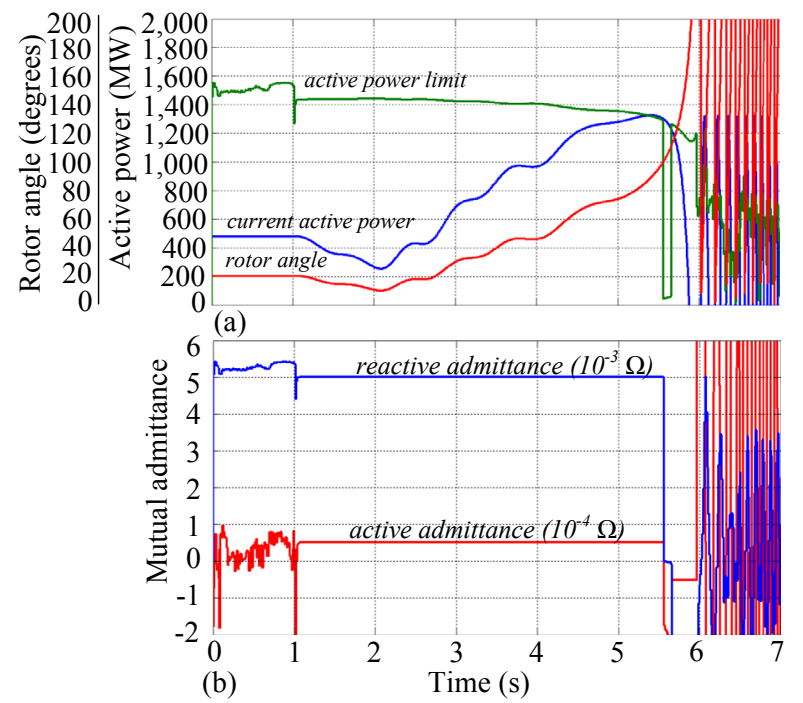

Fig. 2 Generator's power control with monitoring of restrictions on the output power and finishing with an intentional violation of the stability limit: (a) operator observed parameters; and (b) identified admittance (window 1-5.5 s).

(1) The absence of a necessity of total equipping of EPS by measuring means. It is enough to install recorders at the power plants buses only.

(2) Network structure independence. As far as an association with specified network sections is not required.

(3) Informativity. Because the structure of the identified SMA matrix shows the structure of the generators rotor motion including information about an existence of in-phase moving generator groups which stability is under the threat in current network conditions. It allows choosing the vector of regime loading appropriated for current conditions when post emergency stability limits are calculated.

(4) Universality. Because stability margin control is possible as for a transmission grid so for electrical networks with distributed generation.

(5) Availability in the turbine power control loop.

\section{The Result of Method and Technology Efficiency Verification}

\subsection{Verification via Simulation Modeling}

Comparative calculations using the SCS (software 
and computing system) "Mustang" held for Surgut EPS of United Power System of Russia, a complete circuit is shown in Fig. 3, and the equivalent (based on an actual matrix SMA EMF equivalent generators) is shown in Figs. $4 a$ and $4 b$.

Parameters of the initial mode of the relevant matrix SMA EMF generators used for subsequent weighting are shown in Table 1.

Matrix SMA identified on the calculation of the transient process after a disturbance EPS mode. In the calculations with weighting as the infinite buses was selected the large condensing plant (named Reftinskaya GRES) and consistently each of the five generating nodes of the circuit was weighted.

Results of calculations limiting on the stability condition active power generating units from the matrix SMA EMF equivalent generators and complete calculations, the equivalent circuit model of the Surgut power unit controlled EPS are shown in Table 2.

It should be noted that in the general case, the calculation error limit active power generating units from the matrix SMA EMF defined: measurement errors regime parameters, structure and amplitudes mutual motion generator rotor on the interval (window) identification matrix SMA, linearity load model, replacement of group generators with in phase moving rotors in the transition process to one equivalent generator, composition accounted regime restrictions, as well as the reliability of the convergence process solutions of the equations of the steady state in weighting procedures.

\subsection{Verification via Physical Models}

The proposed method was estimated in conditions approximated to actual operating conditions using the digital-analog-physical simulator (electro-dynamic simulator-EDS) of High Voltage Direct Current Power Transmission Research Institute, JSC and the EDS of Novosibirsk State Technical University. Verification of physical and mathematical models

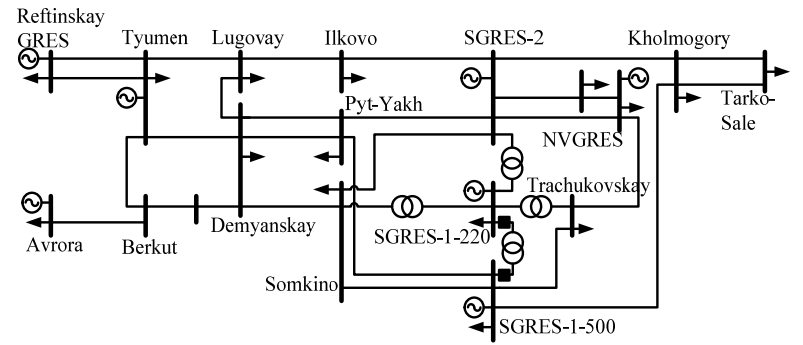

Fig. 3 Scheme $500 \mathrm{kV}$ of the Surgut EPS.

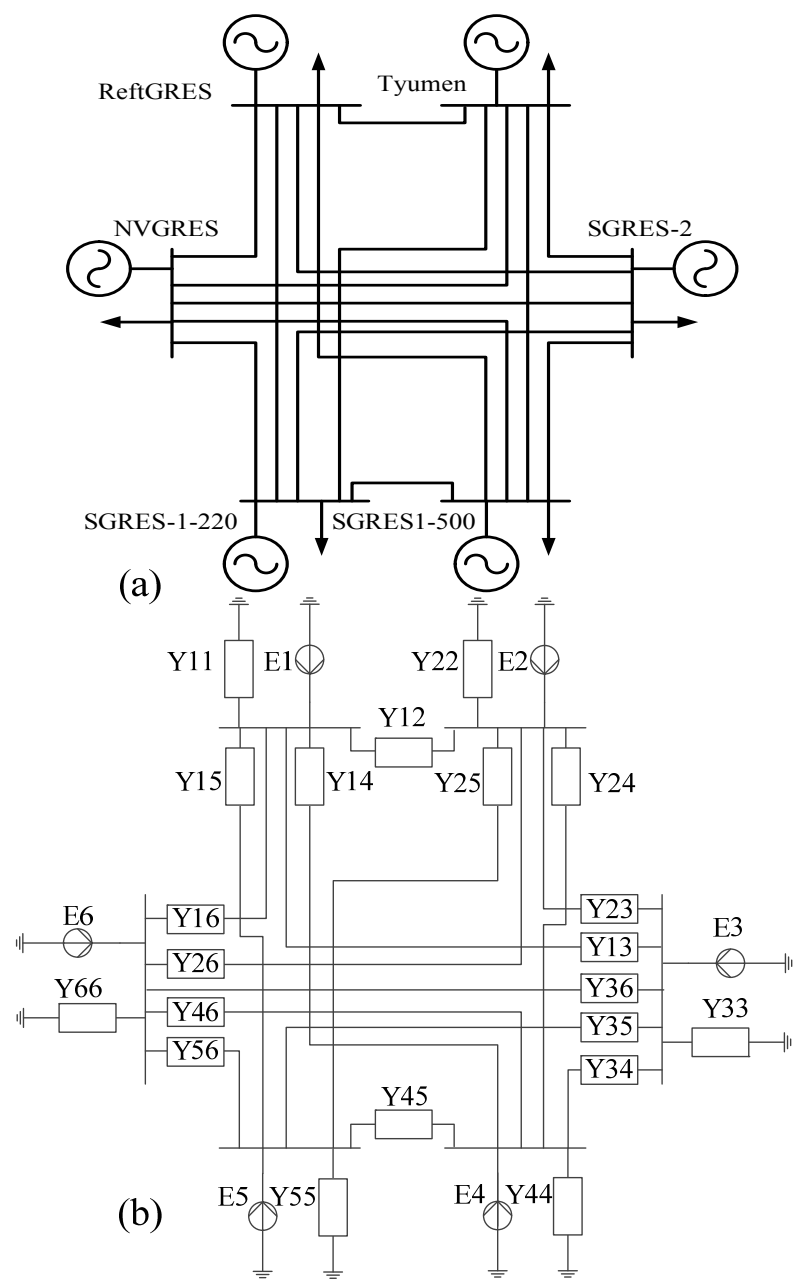

Fig. 4 Equivalent Surgut EPS: (a) equivalent circuit diagram; and (b) equivalent computational scheme.

(created in the Mustang Software) was performed by comparison of process oscillograms.

\subsubsection{Schemes for the Testing}

Schemes of test power systems are presented in the Fig. 5.

\subsubsection{Testing at the Test Center of NSTU}

The electrodynamic model of the NSTU test center is equipped by system of the synchronized 
measurements SMART-WAMS manufactured by CJSC RTSoft for registration the transient mode parameters. Software and hardware complex SMART-WAMS based on multifunctional transducers (MIP-02) allows to organize up to 6 registration points of parameters, the electromechanical transient calculated on $20 \mathrm{~ms}$ interval with a unified timestamp. MIP-02 is connected to the secondary circuits of current and voltage circuits breakers that ensure the measuring point in different parts of the circuit depending on the location of the circuit breaker.

Points synchronized measurements including current and voltage vectors of the positive sequence are denoted by $\triangle$ in Figs. 5a and 5b.

Fig. 6 shows the oscillation of the generator mode parameters by disturbance after autoreclosing of circuit

Table 1 Parameters of the initial regime for weighting based on the relevant matrix SMA EMF generators.

\begin{tabular}{llllll}
\hline $\begin{array}{l}\text { Number } \\
\text { of node }\end{array}$ & Name & $\begin{array}{l}\text { Active power of generator } \\
(\mathrm{W})\end{array}$ & $\begin{array}{l}\text { EMF } \\
(\mathrm{kV})\end{array}$ & $\begin{array}{l}\text { Reactive power of generator } \\
(\mathrm{MVAr})\end{array}$ & $\begin{array}{l}\text { Rotor angle } \\
(\mathrm{rad})\end{array}$ \\
\hline 1 & ReftGRES & 703.4 & 522.0 & 527.6 & 0.0000 \\
2 & Tyumen & $1,203.0$ & 556.1 & 262.2 & 0.2067 \\
3 & SGRES-2 & $4,800.0$ & 546.7 & $1,428.8$ & 0.4947 \\
4 & SGRES-1-220 & $1,165.0$ & 583.3 & 791.9 & 0.1114 \\
5 & NVGRES & 865.0 & 583.5 & 523.5 & 0.3980 \\
6 & SGRES-1-500 & 2,008 & 545.4 & 593.4 & 0.4185 \\
\hline
\end{tabular}

Table 2 Estimated limiting active power generating units.

\begin{tabular}{|c|c|c|c|c|c|}
\hline \multirow{2}{*}{\multicolumn{2}{|c|}{$\begin{array}{l}\text { Power system model } \\
\text { (including AER) }\end{array}$}} & \multirow{2}{*}{$\begin{array}{l}\text { Name nodes } \\
\text { weighting }\end{array}$} & \multicolumn{2}{|c|}{ Limit active power generating units (MW) } & \multirow{2}{*}{$\begin{array}{l}\text { Disagreement regarding the } \\
\text { complete digital model EPS (\%) }\end{array}$} \\
\hline & & & Complete digital model of EPS & Matrix SMA EMF generators & \\
\hline \multirow{10}{*}{$\begin{array}{l}\text { Model } \\
\text { generator } \\
\text { voltage } \\
\text { regulation }\end{array}$} & \multirow{5}{*}{$\begin{array}{l}E=\text { var }, \\
\mathrm{U}_{\mathrm{g}}=\mathrm{const}\end{array}$} & Tyumen & $8,856.3$ & $8,937.4$ & 0.92 \\
\hline & & SGRES-2 & $7,207.5$ & $7,208.8$ & 0.02 \\
\hline & & SGRES-1-220 & $3,633.9$ & $3,598.5$ & 0.97 \\
\hline & & NVGRES & $3,269.0$ & $3,266.9$ & 0.06 \\
\hline & & SGRES-1-500 & $4,411.0$ & $4,413.5$ & 0.06 \\
\hline & \multirow{4}{*}{$\begin{array}{l}E=\text { var, } \\
\mathrm{U}_{\mathrm{g}}=\text { const, } \\
\text { while }\end{array}$} & Tyumen & $5,326.6$ & $5,357.0$ & 0.57 \\
\hline & & SGRES-2 & $6,378.7$ & $6,327.5$ & 0.80 \\
\hline & & SGRES-1-220 & $2,753.1$ & $2,780.3$ & 0.99 \\
\hline & & NVGRES & $2,437.3$ & $2,409.0$ & 1.16 \\
\hline & $Q_{g}<Q_{\max }$ & SGRES-1-500 & $3,588.0$ & $3,588.8$ & 0.02 \\
\hline
\end{tabular}

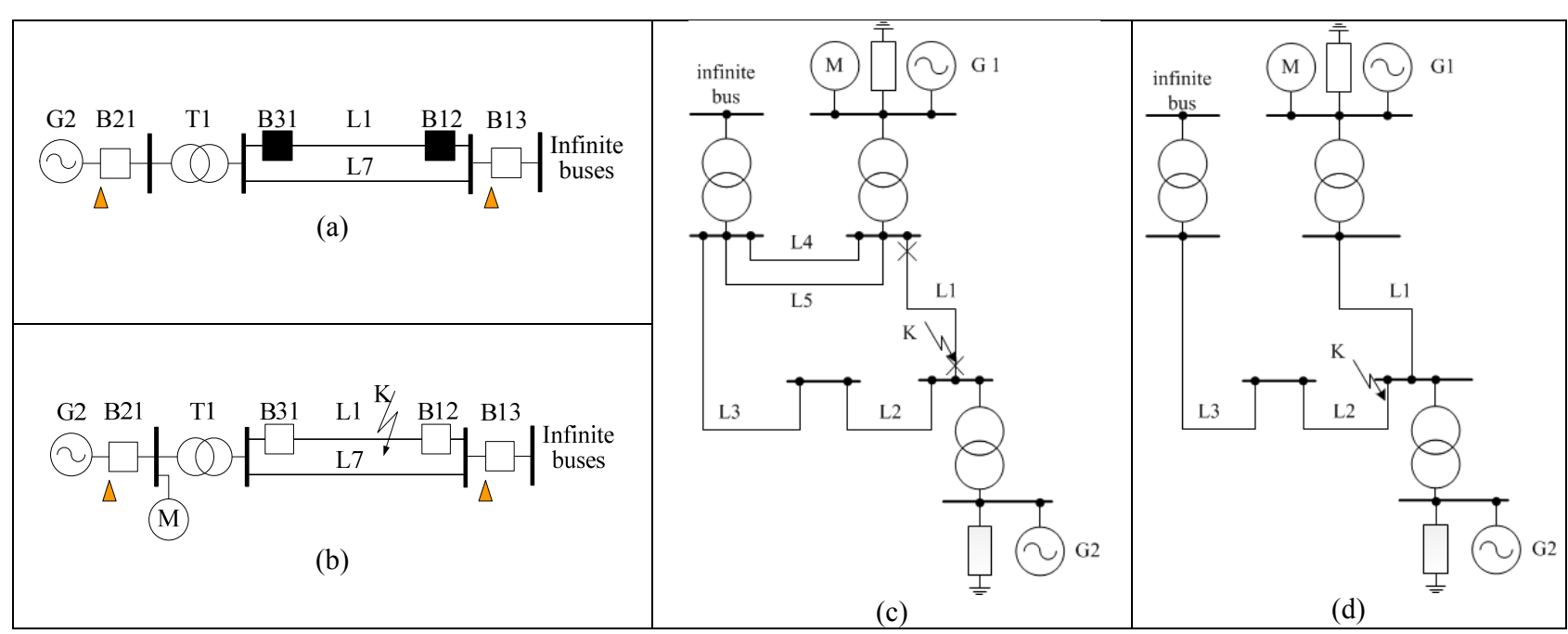

Fig. 5 Schemes of physical models of test power systems: (a), (b) schemes at the test center of NSTU that differ by a type of a loading connected to generator buses; and (c), (d) schemes at the test center of NIIPT with radial and ring topologies. 


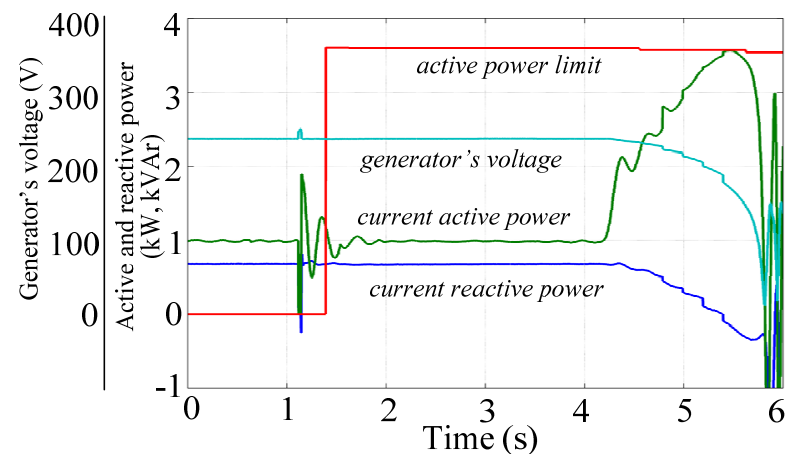

Fig. 6 Change of operation parameters of the generator and determination limit of the output power with subsequent verification.

Table 3 Generator output active power limits (the scheme is presented in Fig. 5a).

\begin{tabular}{llll}
\hline \multirow{2}{*}{$\begin{array}{l}\text { Generator's mode } \\
\text { of reactive power }\end{array}$} & \multicolumn{3}{c}{ The power limit (W) } \\
\cline { 2 - 4 } & $\begin{array}{l}\text { From the } \\
\text { weighting }\end{array}$ & $\begin{array}{l}\text { From the } \\
\text { SMA matrix }\end{array}$ & $\begin{array}{l}\text { From } \\
\text { measurement }\end{array}$ \\
\hline Lines L1, L7 are switched on \\
\hline Overexcitaion & 4,150 & 4,220 & 4,100 \\
Underexcitation & 2,307 & 2,317 & 2,150 \\
\hline Line L1 is switched on & & \\
\hline Overexcitaion & 3,486 & 3,553 & 3,560 \\
Underexcitation & 1,980 & 2,000 & 1,990 \\
\hline
\end{tabular}

breaker B21 (Fig. 5a) with defining the restriction on the generator's output active power and its further test by loading the generator to its hold-off.

Results of assessment of the output active power limit obtained by means of a weighting method and by means of the SMA matrix are presented in Table 3 in compare with measured value.

\subsubsection{Testing at the Test Center of NIIPT}

Measurements of operation parameters were performed by means of the fault recording system developed at the test center of NIIPT. The recording of instantaneous voltages and currents values were performed with the rate of 2,400 samples per second. By using measured values of the current and the voltage of a generator, the active power, the reactive power, effective values of the voltage and the mutual phase were estimated with the period of $30 \mathrm{~ms}$.

In Fig. 7 for the scheme presented in Fig. 5b, in the condition of a tripped two-phase fault at the sending end of the line L2, changes of the active power of the generator during the transient process $(\mathrm{P})$ and the real time estimation of the output power limit of a generator by the terms of static stability by using the SMA matrix are presented.

In Fig. 8a, changes of operation parameters of a generator during the electromechanical process obtained by using the physical model are shown. In Fig. $8 \mathrm{~b}$, a real time estimation of the output power limit of a generator by means of the SMA matrix is presented.

The output active power limits that were obtained by means of a weighting method and by means of a matrix of the SMA using of a physical model are 8.0 and 8.2 $\mathrm{kW}$ respectively.

Results of assessment of the output active power limit that were obtained by means of a weighting method and by means of the SMA matrix in conditions of changes of operation parameters using physical models (for schemes with two generators) are presented in Fig. 9 and Table 4.

Results obtained by using physical models in conditions approximated to actual operating conditions confirm a validity of abstract theorems that are presented in Section 2.

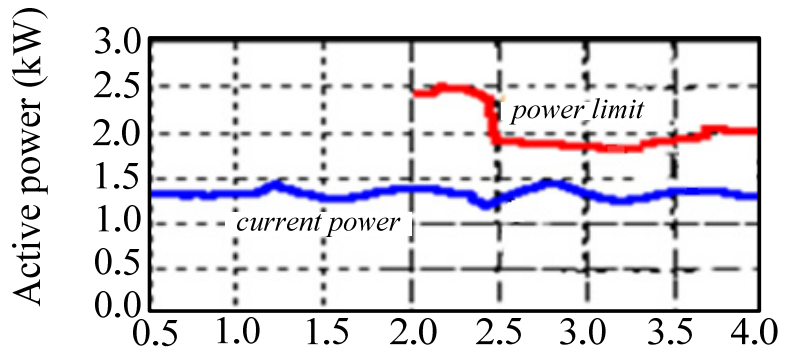

(a)

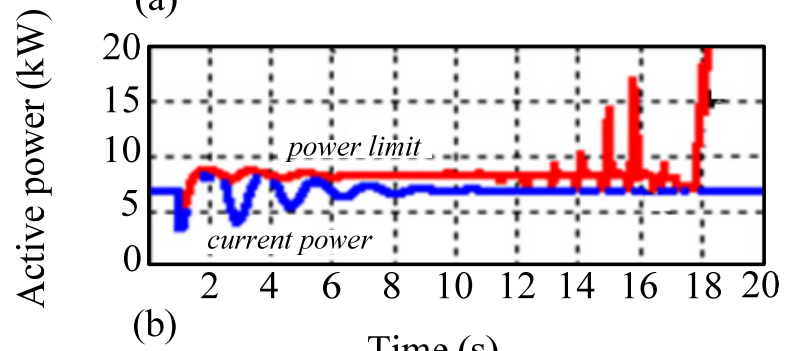

Fig. 7 The current active power $(P)$ and the output active power limit (Pmax) of the generator (the simulation modeling, the window of the identification of the SMA is 2-12 s). 


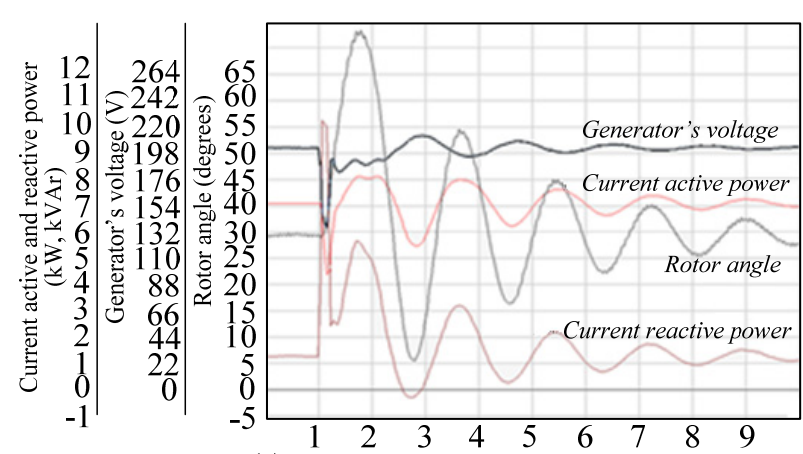

(a)

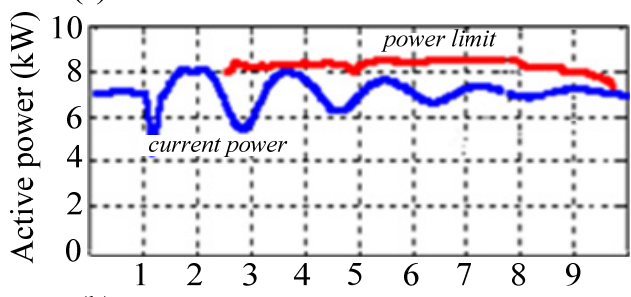

(b)

Time (s)

Fig. 8 The oscillogram and an estimation of the output power limit obtained by using a physical model: (a) changes of operation parameters of generator in the condition of a tripped two-phase fault at the sending end of the line L2; and (b) the current active power $(P)$ and a real time estimation of the output power limit of the generator (Pmax), a window of the SMA identification is 2-8 s.
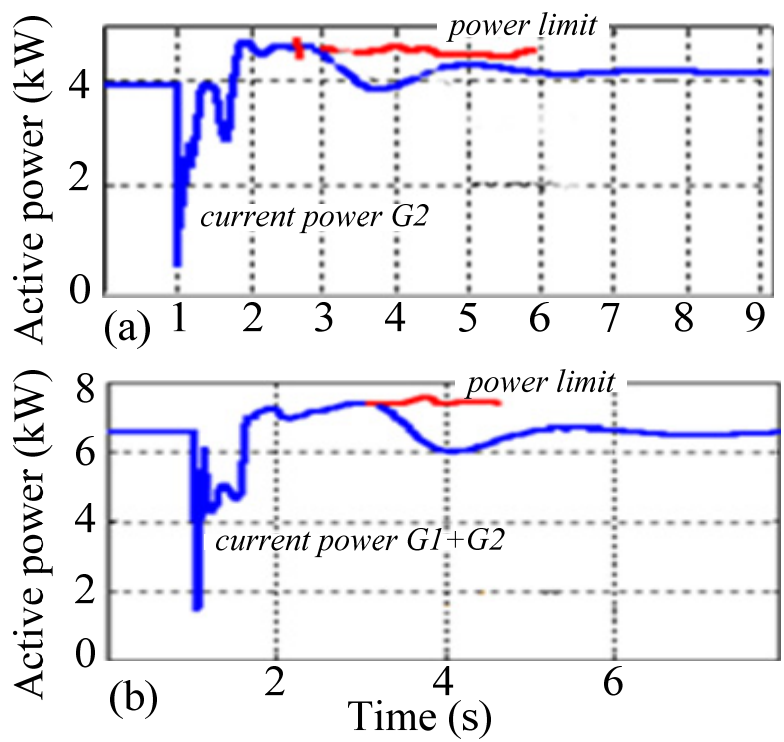

Fig. 9 Assessments of output power limits using a physical model: (a) the output power limit of the generator G2 (Fig. 5c), a window of the SMA identification is 2.5-5.5 s; and (b) the output power limit of an equivalent of the in-phase group of generators that consists of generators G1 and G2 (Fig. 5d), a window of the SMA identification is 2.5-4.5 s.
Table 4 Output power limits of the generator G2 (Fig. 5c) and the equivalent of the in-phase group of generators that consists of generators G1 and G2 (Fig. 5d).

\begin{tabular}{lll}
\hline \multirow{2}{*}{ Scheme } & \multicolumn{2}{c}{ The power limit (W) } \\
\cline { 2 - 3 } & From the weighting & From the SMA matrix \\
\hline Fig. 5c & 4,700 & 4,570 \\
Fig. 5d & 7,450 & 7,400 \\
\hline
\end{tabular}

\section{Conclusions}

It is possible to identify an actual matrix of the SMA (self and mutual admittances) of generator EMFs by using the synchronized (phasor) measurements at generator buses and to get a real time assessment of limit conditions of a power system by the terms of a static stability via matrix of the SMA.

It is advisable to perform a real time monitoring of static stability limits in a post-emergence mode of a power system in two stages: the first stage is a short-term quasi steady mode that comes immediately after the electromechanical transient attenuation; the second stage is long-term post-emergency state. It is useful to get static stability limits at each stage and then use those assessments in the emergency automation.

The proposed technology is focused on the use of real-time and does not require data on the topology, parameters and loads of the electric network.

\section{Acknowledgments}

The authors thank Victor Denisov, Ph.D., associate professor of Automated Electrical Power Systems Department NSTU, Leonid Grashchenkov, Michael Plyuschev, Alexander Nazarenko, specialists Novosibirsk RDO SO UPS, JSC for their invaluable assistance in the preparation of equipment for carrying out experiments on the EPS physical model at the test center of NSTU.

\section{References}

[1] Ayuyev, B. I., Davydov, V. V., and Erokhin, P. I. 2010. "Optimization Model of Limiting Operating Modes of Electric Systems.” Electricity 11: 2-12.

[2] Mohammadi-Ivatloo, B., Shiroei, M., and Parniani, M. 2011. "Online Small Signal Stability Analysis of Multi-machine Systems Based on Synchronized Phasor 
Measurements in Nodes of Its Connection

Measurements." Electric Power Systems Research 81 (10) 1887-96.

[3] Chakrabortty, A., Chow, J. H., and Salazar, A. 2010. “A Measurement-Based Framework for Dynamic Equivalencing of Large Power Systems using WAMS.” In Proceedings of 2010 ISGT Innovative Smart Grid Technologies, 1-8.

[4] Chusovitin, P. V., and Pazderin, A. V. 2013. "Monitoring of Power System Stability Based on a Dynamic Equivalent Determined from Vector Measurements." Electricity 2: 2-10.

[5] Toutoundaeva, D. V., and Fishov, A. G. 2011. "Improvement of the Power System Stability Margin
Control and Standardization Technique." Scientific Herald of Novosibirsk State Technical University 2 (43): 147-60.

[6] Soboleva, M. A., and Fishov, A. G. 2013. "Determining the Limiting Electric Power System Operating Conditions on the Basis Admittances Matrices with Respect to the EMFs of Equivalent Generators." Electricity 8: 9-14.

[7] Fishov, A. G. 2013. The Way of Stock Control the Stability of the Mode of Synchronous Electric Machines Included in the Electricity Network. RU Patent 2,500,061 C2, filed December 02, 2011 and issued November 27, 2013. 